\title{
Effect of rinse cycle softener on water vapour transmission of cotton and polyester
}

\author{
Mehreen Ijaz", Namood-e-Sahar and Zahra Rasheed \\ Department of Home Economics, Lahore College for Women University \\ *Corresponding author's email: mehreenijaz@hotmail.com \\ Citation \\ Mehreen Ijaz, Namood-e-Sahar and Zahra Rasheed. Effect of rinse cycle softener on water vapour transmission \\ of cotton and polyester. Pure and Applied Biology. Vol. 9, Issue 4, pp2435-2440.
}

http://dx.doi.org/10.19045/bspab.2020.90258

\begin{tabular}{llll}
\hline \hline Received: 27/04/2020 & Revised: 26/06/2020 & Accepted: 02/07/2020 & Online First: 24/07/2020 \\
\hline \hline
\end{tabular}

\section{Abstract}

The current paper aims at determining the effect of rinse cycle softener treatment on water transmission behavior of fabrics made with $100 \%$ cotton and polyester. The research work was conducted at Nishat Mills Limited (Dyeing and Finishing Unit). Two groups of samples were formulated. One group was given rinse cycle softener treatment and the other group was not treated with any softener treatment. Both groups were evaluated for their water vapour transmission by following test standard given by American Society for Testing and Materials ASTM E-96 at various washing intervals. The results depicted that rinse cycle treated fabrics had significantly decreased the water vapour transmission through their structure in cotton fabrics with increasing washing intervals. Whereas, no significant difference was observed for polyester fabrics. Moreover, fabrics treated with no softener treatment had increased the water transmission values for both groups. Findings of the study can be beneficial for the consumers to make a decision about the use of fabric softeners in hot climate by considering their change in structure or appearance of fabrics.

Keywords: Cotton; No Softener Treatment; Polyester; Rinse Cycle Softener; Washing Interval; Water Vapour Transmission

\section{Introduction}

This study aims at investigating the effect of locally available rinse cycle fabric softener on water vapour transmission of $100 \%$ Cotton and $100 \%$ polyester after various laundering intervals. A lot of research work has been accomplished to determine water transmission through the clothing structure for better performance of clothes [1]. Water / fluid transmission is highly associated with the moisture present in the surrounding air, movement of air, fabric geometry, fiber type, yarn nature, yarn count and physical properties of the material [2-4].
Water vapor transmission is considered as the rate at which water vapor travels through a textile material [5]. The transfer of moisture (water / vapour) is usually made from wet atmosphere to a dry atmosphere till the equilibrium state [6]. Water transmission depends on fiber-liquid molecular attraction forces that take place on fabric surface and the area of pore distribution in the yarn structure. Moreover, fiber content, type and amount of twist, finish applied over the surface of fabric may also affect the transportation process [4].

Many textile characteristics influence the thermal comfort of an outfit. These 
characteristics include water absorbency, wicking ability, water vapor transmission, heat transfer or air permeability etc [7]. A fabric that carries away moisture in the form of water or vapour and also to increase evaporation of water / liquid contributes to the thermal comfort of a wearer especially during summer season [8]. Various physical properties of textile fibers are greatly affected by water absorbed in their structure such as tensile and tear strength, crease recovery, elasticity, flexibility, rigidity and so on. For example some fabrics are hygroscopic and can readily absorb water vapour in the moist atmosphere as well as desorb water in the dry atmosphere [9]. When water wets the textile fiber, capillary forces shift the liquid into capillaries by spaces present within the fibers during wicking [10]. Wetting action increases with a certain decrease in the surface tension between fabric upper surface and the amount of liquid [11].

Today's consumer is very much concerned with the appearance, feel and hand of the clothing items. There are many laundry aids which are manufactured for this purpose, fabric softener is one of these products designed to satisfy the consumer needs and wants [12].

Fabric softeners were introduced during 1950's and became popular soon. A softener is supposed to increase the fabric pliability by reducing the friction between fabric components. Hair like structure of fiber allows them to move apart from each other. This readily shifting is considered to be responsible for reduction in wrinkling, improved smoothness and handle and easy pressing and ironing of garments [12-14]. These softeners not only aimed to improve the fabric handle but also served as a tool to reduce static clinginess by holding adequate amount of moisture in the fibers to dissipate static charges. Fabric softeners have been extensively used for almost more than fifty years. Silicone based softeners are popular for making soft and smooth fabrics. Cationic based softeners are used after laundering the garments and non-ionic treatments are becoming popular due to their increased compatibility with many chemicals used in various industrial procedures [16]. There are so many advantages associated with these softeners by the manufacturers such as repelling water, fluids and oily stains, decreasing drying time, producing pleasant smell, facilitating ironing, reducing wrinkling and reducing pilling etc $[17,18]$. On the other hand, these softeners are reported to cause yellowing of fabrics, staining, and reducing absorbency [12, 13].

Puchta [19] reported that three types of household softeners are commonly used by the consumers. These include water cycle softeners (used in the washing cycles), rinse cycle softeners (used in final rinse cycle) and dryer sheet softeners (used in the drying cycle). These products contains isopropanol in various concentrations and water content. Isopropanol assists in dissolving cationic agent and role of water is to make sure the dispersion of specific softener in water [15].

Initially rinse cycle softeners were manufactured and presented to the customers followed by wash cycle softeners and dry sheet softeners. The aim of rinse cycle softeners was to make fabric soft in handle and improve its appearance [20]. Rinse cycle softeners are prepared with compounds of dehydrogenated dimethyl ammonium, which have the properties of softening and making the material anti-static [21]. Cotton and polyester are commonly used by the consumers. These consumers usually apply various softeners in order to get an improved and better feel for their garments. For this purpose, the study was focused to investigate the effect of commonly used softener on cotton and polyester fabrics against their water vapour transmission behavior.

\section{Materials and methods}

The study was experimental in nature. The laboratory work was completed at Dyeing and finishing unit of Nishat Mills Limited. The samples were collected from the same 
mill. These samples comprised of $100 \%$ Cotton and $100 \%$ Polyester. The selected fabrics were manufactured by following plain interlacing pattern. Their construction parameters were given in the (Table 1). These collected samples were divided into two groups. Both groups were laundered by following the same test procedure and evaluated for their water vapour transmission. The first group was experimental group which was treated with rinse cycle softener whereas, the other group was taken as control group and no softener treatment was given to it.

Table 1. Construction specifications of collected samples

\begin{tabular}{|c|c|c|c|c|}
\hline Sample & $\begin{array}{c}\text { Fabric weight } \\
\text { (GSM) }\end{array}$ & Yarn count & $\begin{array}{c}\text { Linear Density } \\
\text { (Warp) }\end{array}$ & $\begin{array}{c}\text { Linear Density } \\
\text { (Weft) }\end{array}$ \\
\hline Cotton $100 \%$ & 141 & $110 \times 80$ & 13.45 & 12.56 \\
\hline Polyester $100 \%$ & 152 & $126 \times 70$ & 17.21 & 17.93 \\
\hline
\end{tabular}

Laundering was done by following Monograph M6 [22]. Front-loading automatic washer was used for laundering the specimens under a normal agitation speed of $45 \pm 10 \mathrm{rpm}$. Each washing cycle comprised of washing, rinsing and drying. The temperature was kept at $54 \pm 2{ }^{\circ} \mathrm{C}$ for $11 \pm 1$ minutes. The standardized detergent $(0.1 \mathrm{~g} /$ liter $)$ was added to each cycle. Locally manufactured rinse softener liquefied with water was added to the machine. Spinning cycle, at $1300 \pm 150 \mathrm{rpm}$ for 12 minutes was given to each sample. Every sample was rinsed twice. Then tumble drying at $68 \pm 6^{\circ} \mathrm{C}$ for 90 minutes was done. Control group in which no softener was applied also subjected to the similar test procedure except the addition of a fabric softener. Samples after every five laundering cycles were assessed for their water vapour transmission. A total of twenty launderings were given. Samples were conditioned at $21^{\circ} \mathrm{C} \pm 1^{\circ} \mathrm{C}$ temperature and $65 \% \pm 2 \%$ relative humidity for 24 hours by following standard suggested by American Society for Testing and Materials ASTM-D-1776 [23] prior to testing.

Water transmission of fabrics was evaluated by following ASTM E-96 [24]. A test dish was filled with distilled water to the level of quarter of an inch from the tested sample. It was placed on a flat horizontal surface. Specimen was fixed to the test dish on its top by sticking it in such a way that upper surface of a dish defined the sample area exposed to the vapor pressure inside the test dish. The test continued for 24 hours in a standard testing atmosphere. The dish assemblage was weighted after every three hours to obtain eight data points. Then the numerical data of water vapour transmission rate was obtained.

The rate of water vapor transmission was calculated by following formula:

$\mathrm{WVT}=(\mathrm{G} / \mathrm{t}) / \mathrm{A}$

Where,

$\mathrm{WVT}=$ rate of water vapour transmission

$\mathrm{G}=$ change in weight

$\mathrm{t}=$ time at which change in weight observed

(G)

$\mathrm{G} / \mathrm{t}=$ slope of a straight line

$\mathrm{A}=$ test area (dish mouth)

\section{Results and discussion}

It was observed from the above results (Table 2) that rinse cycle softener decreased the water vapour transmission values for both cotton and polyester fabrics. Whereas the results of no softener treatment depicted an increase in water transmission through these fabrics. One possible reason of decrease in water vapour transmission rate of experimental group was an increase in the fabric mass due to the addition of fabric softener. The similar phenomenon was studied by Whelan et al. [25] that an increase in the fabric surface also increases the resistance against water vapour transmission. 
Table 2. Water vapour transmission of samples

\begin{tabular}{|c|c|c|c|c|c|}
\hline \multirow{2}{*}{ Sample } & \multirow{2}{*}{$\begin{array}{l}\text { Wash } \\
\text { cycle }\end{array}$} & \multicolumn{2}{|c|}{$\begin{array}{c}\text { Rinse cycle softener } \\
\text { Experimental group }\end{array}$} & \multicolumn{2}{c|}{$\begin{array}{c}\text { No softener treatment } \\
\text { Control group }\end{array}$} \\
\cline { 3 - 6 } & Mean & SD & Mean & SD \\
\hline Cotton & 1 & 83.01 & 0.72 & 80.56 & 0.95 \\
\hline & 5 & 80.55 & 0.50 & 81.55 & 0.18 \\
\hline & 10 & 77.58 & 0.23 & 82.21 & 0.60 \\
\hline & 15 & 74.99 & 0.70 & 82.50 & 4.87 \\
\hline & 20 & 73.98 & 0.72 & 84.20 & 0.26 \\
\hline Polyester & 1 & 80.88 & 0.10 & 77.50 & 0.76 \\
\hline & 5 & 80.95 & 0.05 & 78.48 & 0.30 \\
\hline & 10 & 82.80 & 0.21 & 78.87 & 0.42 \\
\hline & 15 & 83.82 & 0.20 & 80.40 & 0.57 \\
\hline & 20 & 84.53 & 0.34 & 81.99 & 0.34 \\
\hline
\end{tabular}

It was also found that rinse cycle softeners had greatly decreased the water absorbency, whereas, dryer softeners had shown insignificant decrease in their absorbency behavior against water $[14,26]$. Williams [20] also observed the fact that increased amount of rinse cycle softener depicted poor water absorbency and water vapour transmission through the tested fabrics. Findings of this study can assist to understand that rinse cycle treatments are not recommended to use in hot weather conditions, because they can make the fabrics feel uncomfortable as they decrease the water vapour transmission.

Many researchers studied the effect of softener treatments on water absorbency of clothes and concluded that treated fabrics took much longer time to sink as compared to untreated even after multiple washings $[12,27]$. In a study it was found that no softener treated fabric was completed absorbed by a drop of water in less time whereas, softener treated fabric took more time to absorb in plain cotton, terry cloth and nylon fabrics. Whereas, no statistical difference was observed among these fabrics when water was showered with pressure [28]. The effect was due to the moisture uptake over the surface of substrate caused by the presence of softener [29].

The effect of fabric components were studied in relation to the application of softeners on garments. It was found that capacity of garments against water and air permeability was decreased [30]. The content of fiber is one of the major reasons in increase or decrease of water vapour transmission through the clothing materials. It is due to the fact that vapors are absorbed by the constituent fibers, transported through them and then finally desorb to the surrounding air [8].

The results indicated that polyester exhibited much higher vapour transmission than cotton (Fig 1). He also suggested that vapour transmission of cotton and polyester blend depends on the ration of blend. The more amount of polyester depicts better efficiency of moisture transmission through the fabric structure. It was observed from the results of the current study that polyester fabric shows less change in the water vapour transmission behavior even with rinse cycle softener. There is an insignificant difference between the values for polyester fabric after repeated number of launderings. So, rinse softener can be used with polyester in hot environment as it does not affect the water vapour transmission.

One possible reason can be the change in fabric dimensions. Many researchers found out that laundering and drying procedures reduce interfiber spaces in fabrics [29]. Increased number of launderings with softener treatment makes the cotton fiber to 
swell due to the absorption of fiber in its structure, it changes the mass, thickness and porosity of the fabric between component fibers. As the rate of porosity and amount of thickness decrease, the fabric becomes less permeable due to low air flow through the structure [31, 32].

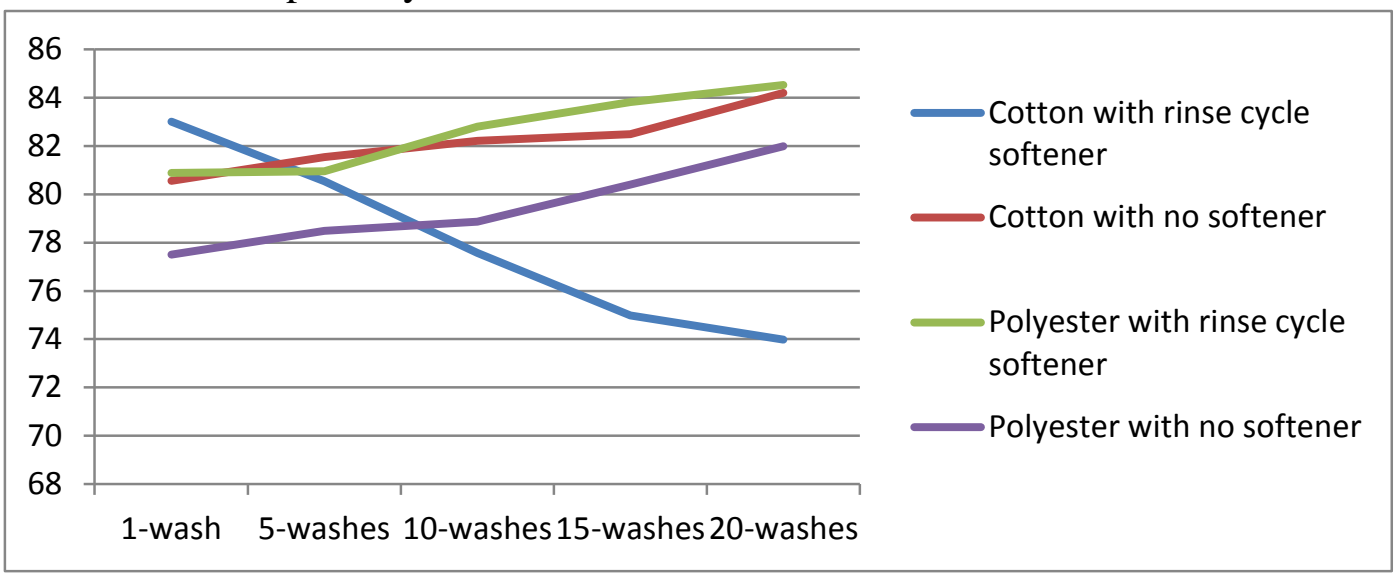

Figure 1. Comparative analysis of cotton and polyester

\section{Conclusion}

This study concludes that rinse cycle treatment given to the tested fabrics decreased the water vapour transmission behavior of fabrics and no softener treatment increased the values of vapour transmission after each washing interval for $100 \%$ cotton fabric. Whereas, rinse cycle treatment did not significantly affect the values for polyester fabric. Findings of the study can be beneficial for the consumers to make a decision about the use of fabric softeners in hot climate by considering their change in structure or appearance of fabrics.

\section{Authors' contributions}

Conceived and designed the experiments: M Ijaz, Performed the experiments: M Ijaz, Analyzed the data: N Sahar, Contributed materials/ analysis/ tools: Z Rasheed, Wrote the paper: M Ijaz.

\section{References}

1. Ding X (2008). Fabric testing. New York: CRC Press, pp 189-224.

2. Berger $X$, \& Sari H (2000). A new dynamic clothing model. Part 1: Heat and mass transfers. Int $J$ Therm Sci 39(6): 673-683.

3. Hepburn CD (1998). The wicking of water through multi-layer fabric assemblies. Doctoral dissertation. University of Leeds.
4. Slater K (1986). Discussion paper the assessment of comfort. J Text I 77(3): 157-171.

5. Kadolph SJ (2007) .Quality assurance for textiles and apparel. Fairchild Publications.

6. Collier BJ, \& Epps HH (1999). Textile Testing and Analysis. Upper Saddle River,NJ: Prentice-Hall, Inc.

7. Taylor MA (1997). Technology of Textile Properties, London: Forbes Publications.

8. Tortora PG \& Collier BJ (1997). Understanding Textiles (5th ed.). Upper Saddle River, NJ: Prentice-Hall, Inc.

9. Ozen I (2012) Multi-layered breathable fabric structures with enhanced water resistance. J Eng Fiber Fabr 7(4): 5769.

10. Hsieh YL (1995). Liquid transport in fabric structures. Text Res J 65(5): 299307.

11. Chatterjee PK (1985). Absorbency.Distributors for the United States and Canada Elsevier Science Publishing Company.

12. Coldwell CA (1968). The effect of fabric softener on white terry cloth laundered in hard water. Doctoral dissertation. Texas Tech University. 
13. Ward HL (1957). Textile softeners for home laundering. J Home Eco 49(2): 122-123.

14. Egan RR (1978). Cationic surface active agents as fabric softeners. $J A M$ Oil Chem Soc 55(1): 118-121.

15. Mullin R (1992). Soaps and detergents. Chem Week 150(2): 24-34.

16. Schindler WD. \& Hauser PJ (2004). Chemical Finishing of Textile. Woodhead Publishing Ltd., Cambridge, England.

17. Murphy AP, \& Kretschmar R (1977). U.S. Patent No. 4,025,444. Washington, DC: U.S. Patent and Trademark Office.

18. Aycock BF, Lawson WH, Moore DR, Rayburn JA, Underwood MP, \& York W (1972). The effect of repeated application of home softeners upon absorbency and other properties of terry towels. Tex Chem Color 4(1): 1621.

19. Puchta R (1984). Cationic surfactants in laundry detergents and laundry aftertreatment aids. J Am Oil Chem Soc 61(2): 367-376.

20. Williams JA (1982). How to choose cationics for fabric softeners. Soap Cos Chem Spec 58(8): 28-32.

21. Baumert KJ, \& Crews PC (1996). Influence of Household Fabric Softeners on Properties of Selected Woven Fabrics. Tex Chem Color 28(4): 75-91

22. AATCC (2013). Monograph M6. Standardization of home laundry test conditions. American Association of Textile Chemists and Colorists: 444446.

23. ASTM (2010). ASTM D1776 Standard practice for conditioning and testing textiles. West Conshohocken, PA: ASTM International. Retrieved from

http://www.astm.org/Standards/D1776 .htm
24. ASTM (2015). ASTM E-96. Standard test method for water vapour transmission of materials. Retrieved from http://www.astm.org/Standards/E96.ht $\mathrm{m}$

25. Whelan ME, MacHattie LE, Goodings AC, \& Turl LH (1955). The Diffusion of Water Vapor through Laminae with Particular Reference to Textile Fabrics: Introduction. Text Res J 25(3): 197198.

26. Robinson K, Gatewood BM \& Chambers IE (1994). AATCC International Conference \& Exhibition. Research Triangle Park, NC: American Association of Textile.

27. Yoon HN, \& Buckley A (1984). Improved comfort polyester: Part I: Transport properties and thermal comfort of polyester/cotton blend fabrics. Text Res J 54(5): 289-298.

28. Gupta D, Kothari VK \& Jhanji Y (2014). Heat and moisture transport in single jersey plated fabrics. J Fibr \& Tex Res 39(1): 115-121.

29. Duru SC, \& Candan C (2013). Effect of repeated laundering on wicking and drying properties of fabrics of seamless garments. Text Res J 83(6): 591-605.

30. Cubric IS, Skenderi Z \& Havenith G (2013). Impact of raw material, yarn and fabric parameters, and finishing on water vapor resistance. Text Res $J$ 83(12): 1215-1228.

31. Şahin UK, \& Cimilli DS (2017). Effects of softener applications on air and water vapor permeability of cotton knitted fabrics produced with different yarns, J Tex App Tek Konf 27(3): 2555.

32. Parthiban M, \& Kumar MR (2007). Effect of Fabric Softener on Thermal Comfort of Cotton and Polyester Fabrics, Indian J Fiber Text 32 (12): 446-452. 